\section{¿Existe asociación entre asma y adiposidad en la población adulta chilena?}

\author{
FANNY PETERMANN-ROCHA ${ }^{1,2, \mathrm{a}, \mathrm{f}}$, CRISTIAN ROCHA ${ }^{3, \mathrm{~b}, \mathrm{f}}$, \\ MARÍA ADELA MARTÍNEZ-SANGUINETTI ${ }^{4, c, f}$, \\ ANA MARÍA LEIVA ${ }^{5, \mathrm{~d}, \mathrm{f}}$, CLAUDIA TRONCOSO-PANTOJA ${ }^{6, \mathrm{a}, \mathrm{f}}$, \\ MARCELO VILLAGRÁN ${ }^{7, c, g}$, NICOLE LASSERRE-LASO ${ }^{8, a, f}$, \\ NATALIA ULLOA ${ }^{9, \mathrm{c}, \mathrm{g}}$, CARLOS CELIS-MORALES $^{2,10, \mathrm{e}, \mathrm{g}}$, \\ EN REPRESENTACIÓN DEL GRUPO ELHOC
}

\section{Association between adiposity and asthma}

Background: In people with asthma, adiposity is associated with more symptoms and less control of the disease whereas on those without asthma adiposity is associated with a higher risk of developing the disease. Aim: To investigate the association between asthma and adiposity markers in Chilean adults. Material and Methods: Data from 5,499 adults older than 15-years-old from the Chilean National Health Survey 20162017 were analyzed. Quintiles of body-mass index (BMI) and waist circumference (WC) were used as a proxy of adiposity. The association between asthma and quintiles of BMI and WC was determined by logistic regression. Results: A higher BMI and WC was associated with a higher odds for asthma. This trend remained significant even after adjusting the models for socio-demographic factors, physical activity and smoking (Odds Ratio [OR] BMI: 1.13 [95\% confidence intervals (CI): 1.04; 1.22], $p<0.01$ and OR WC: 1.15 [95\% IC: 1.06; 1.25], $p<0.01$ ). Conclusions: A higher BMI and WC were positively associated with asthma.

(Rev Med Chile 2019; 147: 733-740)

Key words: Adiposity; Asthma; Obesity; Respiratory Tract Diseases.

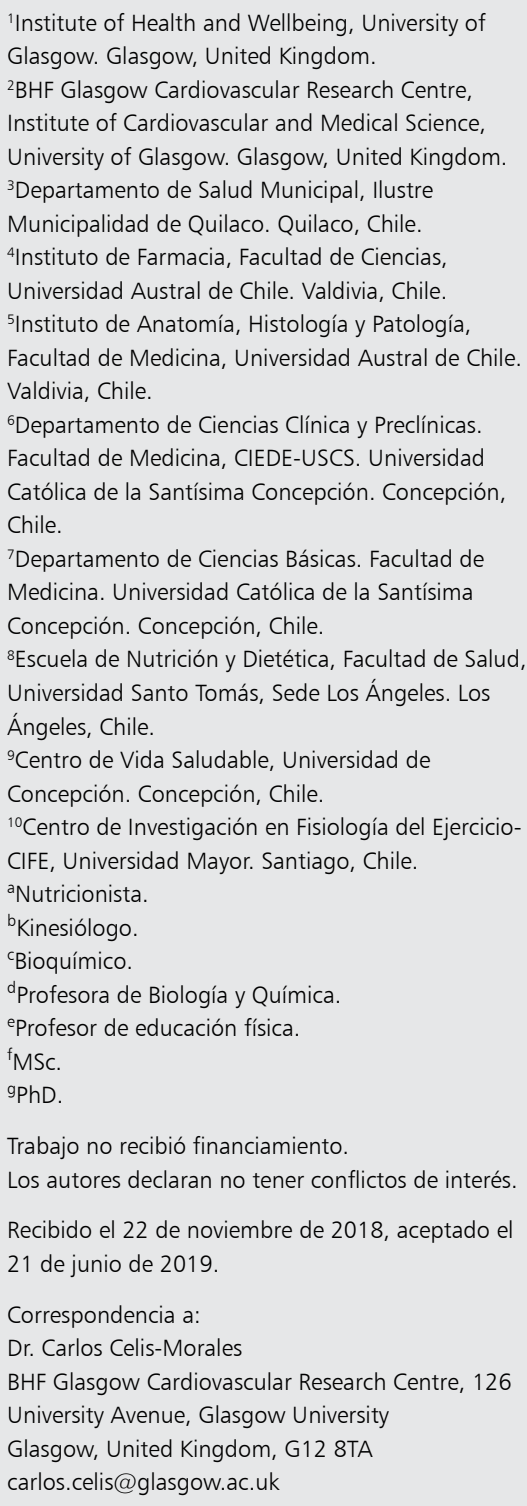

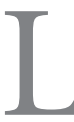

as enfermedades crónicas no transmisibles (ECNT) son enfermedades de larga duración caracterizadas por presentar una lenta progresión y afectar a todos los grupos de edad y regiones en el mundo ${ }^{1}$. Entre los principales tipos de ECNT se identifican cuatro grupos de patologías: las enfermedades cardiovasculares, cáncer, diabetes mellitus tipo 2 y enfermedades respiratorias, entre ellas el asma ${ }^{2}$. El asma es un desorden heterogéneo, complejo, crónico y multifactorial con una fuerte influencia ambiental ${ }^{3}$, caracterizado por presentar períodos de sibilancia, disnea, opresión torácica y/o tos, variando en severidad y frecuencia entre personas ${ }^{4}$. Se estima que 235 millones de individuos padecen asma a nivel mundial y que, a pesar de ser una patología de mayor frecuencia durante la ni$\tilde{n} z^{5}$, en el año 2015 se notificaron 383 mil muertes por asma, principalmente en adultos mayores ${ }^{6}$. 
Pese a que gran parte de la prevalencia de asma puede ser relacionada con la presencia de algunos tipos de alergia, no todos los individuos asmáticos presentan una respuesta alérgica elevada. En este contexto, se han evidenciado y propuesto otros factores de riesgo vinculados con esta patología, entre ellos factores no modificables como el sexo (mayor prevalencia en mujeres) $)^{7,8}$ y la etnia (mayor prevalencia en población afro-americana $)^{3}$; factores genéticos ${ }^{9}$; factores ambientales, como la contaminación ambiental, la higiene durante la infancia o el tipo de trabajo que se realiza; y otros asociados al estilo de vida, como el estrés, la ansiedad y la adiposidad ${ }^{3,10}$.

Estudios longitudinales han evidenciado que, en personas con asma, la obesidad y otros marcadores de adiposidad (como un perímetro de cintura [PC] y porcentaje de grasa corporal elevado) se asocian con un aumento de los síntomas, mayor uso de broncodilatadores, una mayor dificultad para el control de esta patología, así como también una mayor probabilidad de desarrollar asma en personas sin diagnóstico previo ${ }^{11,12}$. Es así como la actual evidencia apunta a hablar de un fenotipo "asmático obeso"13.

Si bien en Chile, según datos de la última Encuesta Nacional de Salud (ENS) 2016-2017, la prevalencia de personas que auto reportaron diagnóstico médico de asma disminuyó (de 6,3\% en el año 2010 a 5,4\% en el año 2017) $)^{14,15}$, la prevalencia de obesidad continúa aumentado (de $27,4 \%$ en el año 2010 a 34,4\% en el año 2017) ${ }^{14,15}$. Pese a esto, pocos trabajos han evaluado la asociación entre obesidad y asma en población adulta chilena ${ }^{16}$, a diferencia de lo que se ha sido reportado en otras poblaciones en el mundo ${ }^{7,17-19}$. Por ende, el objetivo de este estudio fue investigar la asociación entre asma e indicadores de adiposidad en población adulta chilena.

\section{Materiales y Métodos}

La muestra seleccionada incluyó a los participantes de la ENS desarrollada entre los años 2016-2017 (ENS 2016-2017). Esta encuesta correspondió a un estudio de prevalencia realizado en hogares en una muestra nacional, probabilística, estratificada y multietápica de 6.027 personas mayores de 15 años con representatividad nacional, regional y área urbana/rural. Para este estudio, se incluyeron un total de 5.499 participantes que poseían información disponible en relación con su índice de masa corporal (IMC), PC y que respondieron a la pregunta diagnóstico de asma de la ENS 2016-2017. El protocolo de la ENS 2016-2017 fue aprobado por el Comité de Ética de Investigación de la Escuela de Medicina de la Pontificia Universidad Católica de Chile. Todos los participantes firmaron un consentimiento informado ${ }^{15}$.

\section{Mediciones antropométricas}

El peso corporal fue medido a través de una balanza digital y la talla a través de un tallímetro con cartabón incorporado, sin zapatos y utilizando ropa ligera. Con estas mediciones se obtuvo el estado nutricional el cual fue clasificado en base a los puntos de corte del IMC según los criterios de la Organización Mundial de la Salud (OMS) para adultos ${ }^{20}$ : bajo peso: $18,5 \mathrm{~kg} / \mathrm{m}^{2}$, normo peso: $18,5-24,9 \mathrm{~kg} / \mathrm{m}^{2}$; sobrepeso: $25,0-29,9 \mathrm{~kg} /$ $\mathrm{m}^{2}$ y obesidad $\geq 30,0 \mathrm{~kg} / \mathrm{m}^{2}$; y en base a los puntos de corte de valoración específica para el adulto mayor: bajo peso: $<22,9 \mathrm{~kg} / \mathrm{m}^{2}$; normo peso: $23,0-27,9 \mathrm{~kg} / \mathrm{m}^{2}$; sobrepeso: $28,0-31,9 \mathrm{~kg} /$ $\mathrm{m}^{2}$ y obesidad: $\geq 32,0 \mathrm{~kg} / \mathrm{m}^{2(21)}$, mientras que la obesidad central fue definida como un perímetro de cintura $\geq 88 \mathrm{~cm} \mathrm{y} \geq 102 \mathrm{~cm}$ en mujeres y hombres, respectivamente ${ }^{15}$.

\section{Presencia de asma}

La presencia de asma se determinó a través del auto-reporte de cada participante a través de la siguiente pregunta: ¿alguna vez un médico o doctor le ha dicho que tiene o que padece de asma? ${ }^{15}$.

\section{Quintiles de IMC y PC}

El IMC y PC fueron clasificado en quintiles (Q) acorde a los siguientes puntos de corte: Quintiles de corte IMC: Q1: $<24,3 \mathrm{~kg} / \mathrm{m}^{2}$; Q2: 24,3 a 26,9 $\mathrm{kg} / \mathrm{m}^{2}$; Q3: 27,0 a 29,6 kg/m²; Q4: 29,7 a $33,0 \mathrm{~kg} /$ $\mathrm{m}^{2}$; Q5: $\geq 33,1 \mathrm{~kg} / \mathrm{m}^{2}$. Los puntos de corte para quintiles de $\mathrm{PC}$ en fueron Q1: $\leq 82,0 \mathrm{~cm}$; Q2: 82,1 a 90,2 cm; Q3: 90,3 a 97,0 cm; Q4: 97,1 a 105,0 $\mathrm{cm} ; \mathrm{Q} 5: \geq 105,1 \mathrm{~cm}$.

\section{Variables sociodemográficas, de estilo de vida y metabólicas}

Las variables sociodemográficas como edad, sexo, zona geográfica de residencia (urbano/rural), nivel de escolaridad (básica $<8$ años, media: 8 a 12 años, y técnica-universitaria $>12$ años) e 
ingreso económico en tertiles (bajo $<250.000$; medio 250.000 a 450.000 ; alto $>450.000$ pesos chilenos) fueron recolectadas mediante el uso de cuestionarios validados en población nacional. Los datos asociados con los estilos de vida, como el tabaquismo, consumo de frutas y verduras, y horas de sueño diarias ( $<7 \mathrm{~h}, 7-9 \mathrm{~h}$ y $>9 \mathrm{~h}$ ) se obtuvieron mediante la aplicación de cuestionarios y tarjeteros validados en la ENS 2016-2017 ${ }^{15}$. Los niveles de actividad física fueron determinados con el cuestionario "Global Physical Activity Questionnaire" (GPAQv2) 22. Los niveles de sedentarismo fueron determinados mediante el auto-reporte de tiempo destinado a actividades que involucren estar sentado o reclinado durante el tiempo libre o de trabajo a través del mismo cuestionario. Se consideró como punto de corte para inactividad física un gasto energético menor a $<600$ METs/ minutos/semana, según las recomendaciones de la OMS y especificaciones en la guía de análisis de GPAQ v2 $2^{22,23}$.

\section{Análisis estadístico}

Los datos de caracterización de la población estudiada son presentados como promedios con su respectiva desviación estándar (DE), para variables continuas o como proporción para variables categóricas con sus respectivos 95\% intervalos de confianza (95\% IC).

La asociación entre asma y quintiles de adiposidad fue investigada a través de análisis de regresión logística utilizando como grupo de referencia aquellos participantes que se encontraban en el quintil más bajo para IMC o PC (Q1). A su vez, se determinó la tendencia de aumentar el odds ratio para asma en la medida que IMC o $\mathrm{PC}$ incrementaron en 1 quintil. Estos resultados son presentados como odds ratio (OR) con su respectivos 95\% IC.

Todos los análisis fueron ajustados por variables de confusión mediante la utilización de tres modelos estadísticos. Modelo 1 - ajustado por sexo; Modelo 2 - ajustado por Modelo 1, pero también por edad, nivel educacional, zona de residencia (rural/urbana) y región de origen en Chile; y Modelo 3 - ajustado por el Modelo 2, pero también por actividad física, tiempo sedente y tabaquismo.

Para todos los análisis se utilizó el programa STATA SE v14. El nivel de significancia fue definido como $\mathrm{p}<0,05$.

\section{Resultados}

Las características generales de la población estudiada, según presencia o ausencia de asma, se identifican en la Tabla 1. La mayor proporción de los individuos con asma fueron mujeres, vivían en zonas urbanas, poseían un menor nivel educacional, ingresos más bajos junto con un peor auto-reporte de salud y bienestar. Además, poseían una mayor prevalencia de obesidad, obesidad abdominal, diabetes mellitus tipo 2 e hipertensión arterial.

La Tabla 2 evidencia la asociación entre asma y quintiles de IMC. En el Modelo mínimamente ajustado (Modelo 1), los resultados identifican que, por cada incremento en un quintil de IMC, el odds ratio para asma aumentó en un 16\% (OR: 1,16 [95\% IC: 1,01-1,25], p < 0,0001). A pesar de que esta asociación entre IMC y asma disminuyó a $13 \%$ tras ajustar el modelo estadístico por factores sociodemográficos (OR: 1,13 [95\% IC: 1,04-1,22], $\mathrm{p}=0,003$ ) y de estilo de vida (OR: 1,13 [95\% IC: $1,13-1,22], p=0,002)$, esta se mantuvo significativa (Figura 1A).

Finalmente, al evaluar la asociación entre asma y quintiles de PC (Tabla 3), se aprecia una asociación similar a la identificada para IMC, evidenciando que, por cada incremento de un quintil de PC, el odds ratio para asma aumentó en un 18\% en el modelo mínimamente ajustado (OR: 1,18 [95\% IC: $1,10-1,28$ ] $\mathrm{p}<0,0001$ ) y en $15 \%$ en el modelo ajustado por variables sociodemográficas y de estilo de vida (OR: 1,15 [95\% IC: 1,06 - 1,25], $\mathrm{p}=0,001)$ (Figura 1B).

\section{Discusión}

La obesidad es un importante factor de riesgo cardiovascular para el desarrollo de enfermedades crónicas no transmisibles, incluyendo las enfermedades respiratorias. Como ha sido descrito previamente $e^{7,17-19}$, la obesidad es un factor de riesgo no solo para el desarrollo de asma, sino también se asocia a un peor control de dicha patología. En este contexto, un paciente asmático, y que además es obeso, representa un gran desafío clínico debido a la heterogeneidad que presentan ambas patologías 4 .

En este estudio se identificó que, en adultos 
Tabla 1. Características generales de la población chilena según ausencia o presencia de asma

\begin{tabular}{|c|c|c|}
\hline & Sin asma & Con asma \\
\hline \multicolumn{3}{|l|}{ Sociodemográficos } \\
\hline Total, $\mathrm{n}$ & 5.148 & 351 \\
\hline Edad, años & $49,0(19,2)$ & $52,2(20,6)$ \\
\hline $\begin{array}{l}\text { Sexo \% } \\
\text { Mujeres }\end{array}$ & $62,8(61,5 ; 64,2)$ & $72,1(67,1 ; 76,5)$ \\
\hline $\begin{array}{l}\text { Zona \% } \\
\text { Urbana }\end{array}$ & $84,2(83,2 ; 85,2)$ & $83,8(79,5 ; 87,3)$ \\
\hline $\begin{array}{l}\text { Educación \%, (95\% IC) } \\
\text { Básica (< } 8 \text { años) } \\
\text { Media ( } 8-12 \text { años) } \\
\text { Técnica-universitaria (> } 12 \text { años) }\end{array}$ & $\begin{array}{l}23,6(22,4 ; 24,7) \\
54,4(53,0 ; 55,8) \\
22,0(20,9 ; 23,2)\end{array}$ & $\begin{array}{l}34,8(29,9 ; 39,9) \\
45,7(40,5 ; 51,0) \\
19,5(15,7 ; 24,0)\end{array}$ \\
\hline $\begin{array}{l}\text { Ingresos \%, } \\
\text { Bajo } \\
\text { Medio } \\
\text { Alto }\end{array}$ & $\begin{array}{l}36,7(35,3 ; 38,2) \\
30,6(29,2 ; 32,0) \\
32,7(31,2 ; 34,1)\end{array}$ & $\begin{array}{l}50,0(44,3 ; 55,7) \\
24,8(20,2 ; 30,1) \\
25,2(20,6 ; 30,4)\end{array}$ \\
\hline \multicolumn{3}{|l|}{ Antropometría } \\
\hline $\mathrm{IMC}\left(\mathrm{kg} / \mathrm{m}^{2}\right)$ & $28,8(5,5)$ & $30,3(6,6)$ \\
\hline $\begin{array}{l}\text { Estado nutricional }<60 \text { años \% } \\
\text { Bajo peso } \\
\text { Normal } \\
\text { Sobrepeso } \\
\text { Obesidad }\end{array}$ & $\begin{array}{c}3,4(2,9 ; 3,9) \\
26,7(25,5 ; 27,9) \\
35,7(34,4 ; 37,0) \\
34,2(32,9 ; 35,5)\end{array}$ & $\begin{array}{c}3,4(2,0 ; 6,0) \\
21,5(17,5 ; 26,1) \\
33,8(29,0 ; 38,9) \\
41,3(36,2 ; 46,5)\end{array}$ \\
\hline Perímetro de cintura $(\mathrm{cm})$ & $93,7(13,8)$ & $97,1(16,3)$ \\
\hline Obesidad abdominal \% & $35,9(31,0 ; 41,1)$ & $64,1(58,9 ; 69,0)$ \\
\hline \multicolumn{3}{|l|}{ Estilo de vida } \\
\hline Actividad física total (MET/min/día) & $987,3(1.512,5)$ & $1.012,5(1.772,6)$ \\
\hline Prevalencia inactividad física \%, (95\% IC) & $32,0(30,7 ; 33,2)$ & $31,6(27,0 ; 36,7)$ \\
\hline Tiempo sedente (h/día) & $3,14(3,02)$ & $3,32(3,06)$ \\
\hline Consumo de porciones de frutas & $2,03(2,04)$ & $2,20(4,13)$ \\
\hline Número de días que consume frutas & $4,33(2,48)$ & $4,28(2,42)$ \\
\hline Consumo de porciones de verduras & $1,87(1,45)$ & $1,77(1,45)$ \\
\hline Número de días que consume verduras & $5,44(2,10)$ & $5,42(2,10)$ \\
\hline $\begin{array}{l}\text { Tabaquismo \% } \\
\text { Nunca } \\
\text { Exfumador } \\
\text { Fumador ocasional } \\
\text { Fumador regular }\end{array}$ & $\begin{array}{c}47,7(46,4 ; 49,1) \\
23,7(22,5 ; 24,8) \\
6,8(6,2 ; 7,5) \\
21,8(20,7 ; 22,9)\end{array}$ & $\begin{aligned} 50,7 & (45,5 ; 55,9) \\
26,2 & (21,9 ; 31,1) \\
7,7 & (5,3 ; 11,0) \\
15,4 & (12,0 ; 19,6)\end{aligned}$ \\
\hline $\begin{array}{l}\text { Horas de sueño diaria \%, } \\
7-9 \mathrm{~h} \\
<7 \mathrm{~h} \\
>9 \mathrm{~h}\end{array}$ & $\begin{array}{c}7,60(1,54) \\
52,6(51,2 ; 53,9) \\
25,2(24,0 ; 26,4) \\
22,2(21,2 ; 23,4)\end{array}$ & $\begin{array}{c}7,48(1,68) \\
45,6(40,4 ; 50,8) \\
26,8(22,4 ; 31,7) \\
27,6(23,2 ; 32,6)\end{array}$ \\
\hline $\begin{array}{l}\text { Auto-reporte de salud y bienestar } \\
\text { Malo } \\
\text { Regular } \\
\text { Bueno }\end{array}$ & $\begin{array}{c}2,0(1,6 ; 2,4) \\
23,9(22,7 ; 25,0) \\
74,1(72,9 ; 75,3)\end{array}$ & $\begin{array}{c}5,1(3,2 ; 8,0) \\
32,2(27,5 ; 37,3) \\
62,7(57,5 ; 67,6)\end{array}$ \\
\hline \multicolumn{3}{|l|}{ Metabólicas \% } \\
\hline Síndrome metabólico & $45,1(43,4 ; 46,8)$ & $45,1(38,9 ; 51,5)$ \\
\hline Diabetes mellitus tipo 2 & $16,9(15,8 ; 18,0)$ & $19,6(15,7 ; 24,3)$ \\
\hline Hipertensión arterial & $35,6(34,3 ; 36,9)$ & $45,7(40,6 ; 51,0)$ \\
\hline
\end{tabular}

Datos son presentados como promedio y desviación estándar para variables continuas, y como proporción con sus respectivo 95\% IC para variables categóricas. 
Tabla 2. Asociación entre asma y quintiles de índice de masa corporal en población chilena

\begin{tabular}{|c|c|c|c|c|c|c|c|}
\hline & & $\mathbf{Q u}$ & tiles de índi & de masa & poral & & \\
\hline & Quintil 1 & Quintil 2 & Quintil 3 & Quintil 4 & Quintil 5 & Tendencia & Valor $\mathbf{p}$ \\
\hline Modelo 1 & $\begin{array}{l}1,00 \\
\text { (Ref.) }\end{array}$ & $\begin{array}{c}1,00 \\
(0,69 ; 1,47)\end{array}$ & $\begin{array}{c}1,12 \\
(0,77 ; 1,61)\end{array}$ & $\begin{array}{c}1,25 \\
(0,87 ; 1,79)\end{array}$ & $\begin{array}{c}1,77 \\
(1,27 ; 2,49)\end{array}$ & $\begin{array}{c}1,16 \\
(1,01 ; 1,25)\end{array}$ & $<0,0001$ \\
\hline Modelo 2 & $\begin{array}{l}1,00 \\
\text { (Ref.) }\end{array}$ & $\begin{array}{c}0,98 \\
(0,67 ; 1,44)\end{array}$ & $\begin{array}{c}1,04 \\
(0,71 ; 1,50)\end{array}$ & $\begin{array}{c}1,14 \\
(0,79 ; 1,64)\end{array}$ & $\begin{array}{c}1,62 \\
(1,15 ; 2,28)\end{array}$ & $\begin{array}{c}1,13 \\
(1,04 ; 1,22)\end{array}$ & 0,003 \\
\hline Modelo 3 & $\begin{array}{r}1,00 \\
\text { (Ref.) }\end{array}$ & $\begin{array}{c}0,99 \\
(0,68 ; 1,45)\end{array}$ & $\begin{array}{c}1,05 \\
(0,72 ; 1,52)\end{array}$ & $\begin{array}{c}1,16 \\
(0,80 ; 1,67)\end{array}$ & $\begin{array}{c}1,62 \\
(1,15 ; 2,29)\end{array}$ & $\begin{array}{c}1,13 \\
(1,04 ; 1,22)\end{array}$ & 0,002 \\
\hline
\end{tabular}

Datos presentados como OR y sus respectivos intervalos de confianza ( $95 \%$ IC). Ref.: el grupo de referencia fue asignado a personas que pertenecían al quintil más bajo para IMC (Q1: $\left.<24,3 \mathrm{~kg} / \mathrm{m}^{2}\right)$. Un valor mayor a 1 indica que hay un mayor riesgo de desarrollar asma en personas pertenecientes a los distintos quintiles para IMC. Los modelos fueron ajustados por: Modelo 1 - ajustado por sexo; Modelo 2 - ajustado por Modelo 1, pero también por edad, nivel educacional, zona de residencia (rural/ urbana) y región de origen en Chile; Modelo 3 - ajustado por el Modelo 2, pero también por actividad física, tiempo sedente y tabaquismo.

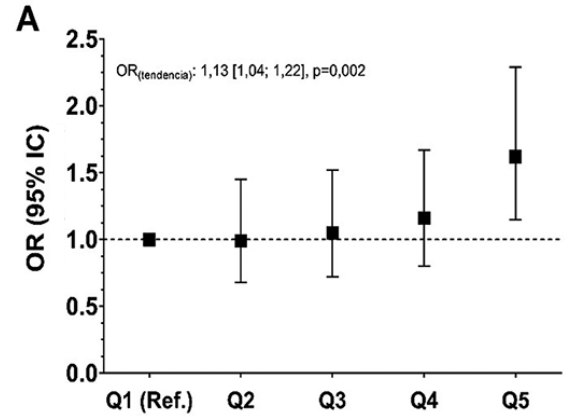

Quintiles de índice de masa corporal

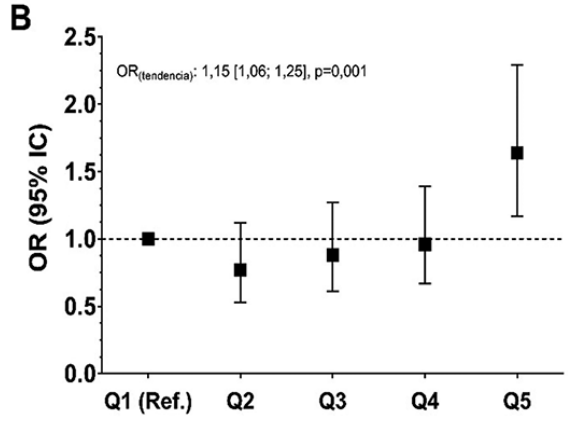

Quintiles de perímetro de cintura

Figura 1. Asociación entre asma y quintiles de índice de masa corporal y perímetro de cintura en población chilena. Datos presentados como odds ratio (95\% IC) para asma según nivel de adiposidad (IMC y PC). La Figura A clasificó a los grupos en base a quintiles de IMC y la Figura B en base a quintiles de PC (Modelo 3). En ambos grupos se utilizó como grupo de referencia aquellas personas en el Q1. Los puntos de corte para quintiles de IMC $\left(\mathrm{kg} / \mathrm{m}^{2}\right)$ fueron Q1: $<24,3 \mathrm{~kg} / \mathrm{m}^{2} ; \mathrm{Q} 2: 24,3$ a 26,9 kg/ $\mathrm{m}^{2}$; Q3: 27,0 a 29,6 kg/m²; Q4: 29,7 a $33 \mathrm{~kg} / \mathrm{m}^{2} ; \mathrm{Q} 5: \geq 33,1 \mathrm{~kg} / \mathrm{m}^{2}$. Los puntos de corte para quintiles de PC en fueron Q1: $\leq 82,0 \mathrm{~cm}$; Q2: 82,1 a 90,2 cm; Q3: 90,3 a 97,0 cm; Q4: 97,1 a 105,0 cm; Q5: $\geq 105,1 \mathrm{~cm}$.

Tabla 3. Asociación entre asma y quintiles de perímetro de cintura en población chilena

\begin{tabular}{|lccccccc|}
\hline & \multicolumn{9}{c}{ Quintiles de perímetro de cintura } & \multicolumn{2}{c|}{ Quintil 1 } & Quintil 2 & Quintil 3 & Quintil 4 & Quintil 5 & Tendencia & Valor p \\
Modelo 1 & 1,00 & 0,81 & 0,93 & 1,10 & 1,84 & 1,18 & $<0,0001$ \\
& (Ref.) & $(0,56 ; 1,18)$ & $(0,64 ; 1,33)$ & $(0,77 ; 1,57)$ & $(1,34 ; 2,54)$ & $(1,10 ; 1,28)$ & \\
Modelo 2 & 1,00 & 0,76 & 0,86 & 0,95 & 1,63 & 1,15 & 0,001 \\
& (Ref.) & $(0,52 ; 1,11)$ & $(0,59 ; 1,24)$ & $(0,66 ; 1,37)$ & $(1,17 ; 2,28)$ & $(1,06 ; 1,25)$ & \\
Modelo 3 & 1,00 & 0,77 & 0,88 & 0,96 & 1,64 & 1,15 & 0,001 \\
& (Ref.) & $(0,53 ; 1,12)$ & $(0,61 ; 1,27)$ & $(0,67 ; 1,39)$ & $(1,17 ; 2,29)$ & $(1,06 ; 1,25)$ & \\
\hline
\end{tabular}

Datos presentados como OR y sus respectivos intervalos de confianza ( $95 \%$ IC). Ref.: el grupo de referencia fue asignado a personas que pertenecían al quintil más bajo para PC (Q1: $\leq 82,0 \mathrm{~cm})$. Un valor mayor a 1 indica que hay un mayor riesgo de desarrollar asma en personas pertenecientes a los distintos quintiles para PC. Los modelos fueron ajustados por: Modelo 1 - ajustado por sexo; Modelo 2 - ajustado por Modelo 1, pero también por edad, nivel educacional, zona de residencia (rural/ urbana) y región de origen en Chile; Modelo 3 - ajustado por el Modelo 2, pero también por actividad física, tiempo sedente y tabaquismo. 
chilenos, en la medida que incrementaron los quintiles de IMC o PC (obesidad y obesidad abdominal), aumentó el odds ratio para asma. Si consideramos que Chile es un país que presenta una gran prevalencia de obesidad en todos los grupos de población y que, a pesar de ser el asma reconocido como una de las enfermedades crónicas de mayor prevalencia durante la infancia, $45,5 \%$ de los ingresos por asma bronquial en los servicios de salud durante el año 2010 correspondieron a adultos $\geq 20$ años (12,7\% mayores de 65 años) ${ }^{24}$, los datos de este estudio son muy relevantes y refuerzan la necesidad de implementar políticas para mejorar el estilo de vida de nuestra población, más aún con el aumento exponencial de adultos mayores, los que están alcanzando una etapa de envejecimiento con una mayor prevalencia de patologías comunes y posibles de prevenir, como es el caso de la obesidad y, en algunas personas, el asma.

Estudios internacionales, en diferentes poblaciones, también han corroborado la asociación entre asma y obesidad ${ }^{7,17-19}$. To y cols. evidenciaron que mujeres japonesas con un IMC $\geq 25 \mathrm{~kg} / \mathrm{m}^{2}$, presentaban un peor control del asma $(\mathrm{p}=0,008)^{7}$. En Estado Unidos de Norteamérica, el estudio longitudinal de seguimiento a una cohorte de enfermeras (The Nurses' Health Study II), corroboró que el IMC posee una relación fuerte, independiente y positiva con el desarrollo de asma, concluyendo el estudio que, al estar estas dos patologías fuertemente relacionadas, el aumento de la prevalencia de obesidad a nivel mundial podría explicar el incremento de la prevalencia de asma ${ }^{17}$. En el caso de Chile, la asociación entre asma y obesidad ha sido principalmente estudiada en población infantil. Vidal y cols. identificaron en 219 niños asmáticos que aquellos que poseían sobrepeso u obesidad poseían una proporción significativamente mayor de asma no controlada en comparación a aquellos asmáticos con estado nutricional normal ${ }^{25}$. De manera similar, Brockmann y cols. evidenciaron que los niños asmáticos son más obesos que sus pares y que el aumento de síntomas como tos, dificultad respiratoria y mayor cansancio durante la realización de actividad física en niños asmáticos, podría ser una de las razones por la cual estos practican menos actividad física ${ }^{26}$, lo que a su vez fomentaría la mantención de la obesidad. En otras palabras, el asma fue asociado a obesidad en forma independiente de los niveles de actividad física.
Finalmente, pese a que diferentes mecanismos biológicos y no biológicos han sido propuestos para explicar la relación existente entre asma y obesidad, aún no está claro si el papel de la obesidad sería inducir el desarrollo de asma o actuar como una comorbilidad ${ }^{4,13}$. Lo que sí está claro es que el aumento de la masa grasa en el abdomen y en la pared torácica reduce la capacidad residual pulmonar, lo que trae como consecuencia la disminución del músculo liso de la vía aérea ${ }^{27}$. Esto, sumado al patrón respiratorio que adoptan las personas con obesidad (aumento de la frecuencia respiratoria y disminución del volumen del flujo), predispone a presentar una mayor hiperreactividad de la vía aérea frente a agentes inflamatorios ${ }^{27}$.

\section{Fortalezas y limitaciones}

Entre las fortalezas de este estudio se encuentra la representatividad nacional de la población escogida, así como también el uso de diferentes factores de confusión asociados con el estilo de vida (no modificables y modificables) lo cual favoreció la interpretación de los resultados. Sin embargo, entre las principales limitaciones se identifican la determinación de la presencia de asma de manera auto-informada y no a través de la confirmación diagnóstica por un médico o cuestionario de signos y síntomas como ha sido reportado en otras poblaciones ${ }^{27,28}$. Esto también explicaría el por qué la prevalencia auto reportada de asma disminuyó según datos de la última ENS 2016-2017, ya que, además de existir una gran cantidad de casos aún no diagnosticados o subdiagnosticados de la patología, estos datos podrían estar sesgados al ser solo un auto reporte. Además, la ENS 2016-2017 carece de un diseño de seguimiento longitudinal, por lo que no permite establecer una relación de causalidad entre asma y adiposidad, solo permite establecer una posible asociación.

\section{Conclusión}

El aumento en los quintiles de IMC y PC (obesidad y obesidad abdominal) fue positivamente asociado con asma. Considerando el dramático aumento en la prevalencia de obesidad en Chile en los últimos 40 años $^{29}$, y que se espera que estas cifras sigan incrementando ${ }^{30}$, los resultados de este estudio confirman la importancia de generar nuevas políticas públicas orientadas a disminuir 
la epidemia de la obesidad, no solamente para disminuir la prevalencia de otras patologías comunes, como diabetes mellitus tipo 2 e hipertensión arterial, sino también como una posible medida destinada a disminuir la prevalencia de asma en nuestro país.

Agradecimientos: Se agradece de manera especial a todos los participantes de la ENS 20162017, al equipo profesional de la Escuela de Salud Pública, de la Facultad de Medicina de la Pontificia Universidad Católica de Chile, quienes desarrollaron y aplicaron la Encuesta Nacional de Salud y al Ministerio de Salud del Gobierno de Chile.

\section{Referencias}

1. WHO. Enfermedades no transmisibles. World Health Organization. 2018; Available: http://www.who.int/es/ news-room/fact-sheets/detail/noncommunicable-diseases. [Consultado el 10 de noviembre de 2018].

2. Gebremariam LW, Chiang C, Yatsuya H, Hilawe EH, Kahsay AB, Godefay H, et al. Non-communicable disease risk factor profile among public employees in a regional city in northern Ethiopia. Sci Rep 2018; 8 (1): 9298.

3. Toskala E, Kennedy DW. Asthma risk factors. Int Forum Allergy Rhinol 2015; 5 Suppl 1: S11-6.

4. García-Río F, Álvarez-Puebla MJ, De Esteban I, Barranco P, Olaguibel JM. Obesity and asthma. Key clinical questions. J Investig Allergol Clin Immunol 2018; 0.

5. WHO. ASMA. World Health Organisation.Available: http://www.who.int/respiratory/asthma/es/.

6. WHO. 10 datos sobre el asma. World Health Organisation. 2017;Available: http://www.who.int/features/factfiles/asthma/es/. [Consultado el 10 noviembre de 2018].

7. To M, Hitani A, Kono Y, Honda N, Kano I, Haruki K, et al. Obesity-associated severe asthma in an adult Japanese population. Respir Investig 2018; 56 (6): 440-7.

8. Hancox RJ, Milne BJ, Poulton R, Taylor DR, Greene JM, McLachlan CR, et al. Sex differences in the relation between body mass index and asthma and atopy in a birth cohort. Am J Respir Crit Care Med 2005; 171 (5): 440-5.

9. Hallstrand TS, Fischer ME, Wurfel MM, Afari N, Buchwald D, Goldberg J. Genetic pleiotropy between asthma and obesity in a community-based sample of twins. J Allergy Clin Immunol 2005; 116 (6): 1235-41.

10. Frieri M, O’Connor M, Nassef M. Asthma, stress, and depression in women. Allergy Asthma Proc 2015; 36 (4):
256-61.

11. Ali Z, Ulrik CS. Obesity and asthma: a coincidence or a causal relationship? A systematic review. Resp Med 2013; 107 (9): 1287-300.

12. Forno E, Acosta-Pérez E, Brehm JM, Han YY, Álvarez $\mathrm{M}$, Colon-Semidey A, et al. Obesity and adiposity indicators, asthma, and atopy in Puerto Rican children. Allergy Asthma Proc. 2014;133(5):1308-14, 14.e1-5.

13. Celebi Sozener Z, Aydin O, Mungan D, Misirligil Z. Obesity-asthma phenotype: Effect of weight gain on asthma control in adults. Allergy Asthma Proc 2016; 37 (4): 311-7.

14. MINSAL. Encuesta Nacional de Salud 2009-2010. Chile: Ministerio de Salud; 2010.

15. MINSAL. Encuesta Nacional de Salud 2016-2017: primeros resultados. Ministerios de salud, Gobierno de Chile. 2017; Available: http://www.minsal.cl/wp-content/uploads/2017/11/ENS-2016-17_PRIMEROS-RESULTADOS.pdf.[Consultado el 30 de octubre de 2018].

16. Bustos $\mathrm{P}$, Amigo $\mathrm{H}$, Oyarzún $\mathrm{M}$, Rona RJ. Is there a causal relation between obesity and asthma? Evidence from Chile. Int J Obes (Lond) 2005; 29 (7): 804-9.

17. Camargo CA, Jr, Weiss ST, Zhang S, Willett WC, Speizer FE. Prospective study of body mass index, weight change, and risk of adult-onset asthma in women. Arch Intern Med 1999; 159 (21): 2582-8.

18. Tang M, Henderson RJ, Holbrook JT, Que LG, Mathews AM, Wise RA, et al. Does Obesity Increase Respiratory Tract Infections in Patients with Asthma? J Allergy Clin Immunol Pract 2019; 7 (3): 954-61.

19. Del-Río-Navarro BE, Fanghanel G, Berber A, Sanchez-Reyes L, Estrada-Reyes E, Sienra-Monge JJ. The relationship between asthma symptoms and anthropometric markers of overweight in a Hispanic population. J Investig Allergol Clin Immunol 2003; 13 (2): 118-23.

20. WHO. Obesity: preventing and managing the global epidemic. World Health Organization. 2000; Disponible en: http://www.who.int/nutrition/publications/obesity/ WHO_TRS_894/en/. [Consultado el 10 de noviembre de 2018].

21. OPS. Parte 1: Módulos de Valoración clínica. Módulo 5: Valoración Nutricional del Adulto Mayor. Organización Panamericana de la Salud. 2003.

22. WHO. Global Physical Activity Questionnaire: GPAQ version 2.0. World Health Organization. 2009;Disponible en: http://www.who.int/chp/steps/resources/ GPAQ_Analysis_Guide.pdf. [Consultado el 10 de noviembre de 2018].

23. WHO. Global recommendations on physical activity for health. World Health Organization. 2010;Disponible en: http://www.who.int/dietphysicalactivity/factsheet_re- 
commendations/en/. [Consultado el 10 de noviembre de 2018].

24. DEIS. Ingreso por asma bronquial, según grupo etario. Departamento de Estadísticas e Información de Salud 2010;Avilable: https://reportesdeis.minsal.cl/REM/2010/ INGRESOS_ASMA_EDAD/INGRESOS_ASMA_EDAD. aspx. [Consultado el 15 de noviembre de 2018].

25. Vidal GA, Escobar CAM, Ceruti DE, Henríquez YMT, Medina R ME. Impacto del sobrepeso y la obesidad en el asma infantil. Rev Chil Enf Resp 2012; 28: 174-81.

26. Brockmann VP, Caussade LS, Holmgren PNL, Prado $\mathrm{AF}$, Reyes MB, Viviani GP, et al. Actividad física y obesidad en niños con asma. Rev Chil Pediatr 2007; 78: 482-8.

27. Jobim Benedetti F, Lúcia Bosa V, Mariante Giesta J,
Bueno Fischer G. Anthropometric indicators of general and central obesity in the prediction of asthma in adolescents; central obesity in asthma. Nutr Hosp 2015; 32 (6): 2540-8.

28. Shin B, Cole SL, Park SJ, Ledford DK, Lockey RF. A new symptom-based questionnaire for predicting the presence of asthma. J Investig Allergol Clin Immunol 2010; 20 (1): 27-34.

29. Celis-Morales C, Leiva A, Martínez MA, Durán E, Labraña AM, Petermann F, et al. Aumento del índice de masa corporal durante las últimas cuatro décadas en la población chilena: de la desnutrición a la obesidad. Rev Med Chile 2017; 145: 1363-4.

30. OECD. Obesity Update 2017. Organization for Economic Co-operation and Development. 2017. 